\title{
The role of Fischer-Tropsch catalysis in Jovian subnebular chemistry
}

\author{
O. Mousis ${ }^{1}$, Y. Alibert ${ }^{2}$, Y. Sekine ${ }^{3}$, S. Sugita ${ }^{4}$, and T. Matsui ${ }^{4}$ \\ 1 Observatoire de Besançon, CNRS-UMR 6091, 41bis avenue de l'Observatoire, BP 1615, 25010 Besançon Cedex, France \\ e-mail: 0livier.Mousis@obs-besancon.fr \\ 2 Physikalisches Institut, University of Bern, Sidlerstrasse 5, 3012 Bern, Switzerland \\ 3 Department of Earth \& Planetary Science, Graduate School of Science, University of Tokyo, 7-3-1 Bunkyo, Tokyo 113-0033, Japan \\ ${ }^{4}$ Department of Complexity Science \& Engineering, Graduate School of Frontier Science, University of Tokyo, 5-1-5 Kashiwanoha, \\ Kashiwa, Chiba 227-8562, Japan
}

Received 1 February 2006 / Accepted 4 August 2006

ABSTRACT

\begin{abstract}
We examine the production of methane via Fischer-Tropsch catalysis in an evolving turbulent model of the Jovian subnebula and its implications for the composition of satellitesimals produced in situ. We show that there is a catalytically-active region in the Jovian subnebula from 65 Jupiter radii that moves inwards with time. The pressure range in this region is about $10^{-4}$ to $10^{-3}$ bar and implies that, if transport processes and the cooling of the subnebula are not considered, $\mathrm{CO}$ and $\mathrm{CO}_{2}$ are entirely converted into $\mathrm{CH}_{4}$ via Fischer-Tropsch catalysis in about $10^{1}-10^{2}$ and $10^{3}-10^{4}$ years, respectively. On the other hand, the comparison of the chemical conversion times with the viscous timescale of the subdisk in the catalytically-active region implies that only $\mathrm{CO}$ can be fully converted into $\mathrm{CH}_{4}$, the conversion of $\mathrm{CO}_{2}$ thus being restricted to a limited production of $\mathrm{CH}_{4}$. Moreover, the time required by the Jovian subnebula to cool down from the optimal temperature for Fischer-Tropsch catalysis to the condensation temperature of ices is at least two orders of magnitude higher than the viscous timescale. This implies that any $\mathrm{CH}_{4}$ produced in the catalytically-active zone will be accreted onto Jupiter long before being incorporated into the forming ices. We then conclude that in an evolving turbulent subnebula, even if Fischer-Tropsch catalysis is active, it has no influence on the composition of the forming satellitesimals that will ultimately take part in the formation of regular icy satellites, in opposition to what has been expected from stationary models.
\end{abstract}

Key words. planets and satellites: formation - solar system: formation

\section{Introduction}

Fischer-Tropsch catalysis, which converts $\mathrm{CO}$ or $\mathrm{CO}_{2}$ and $\mathrm{H}_{2}$ into $\mathrm{CH}_{4}$ on the surface of transition metals such as iron and nickel, is believed to play important roles in astrophysical environments. In addition to producing large quantities of water in some circumstellar envelopes (Willacy 2004), it has also been shown to be a means of producing hydrocarbons in oxygen-rich regions such as the protosolar nebula (Kress \& Tielens 2001). Laboratory experiments to determine $\mathrm{CH}_{4}$ reaction rates under a hydrogen-dominated gas-phase and at low-pressure conditions have been recently conducted by Sekine et al. (2005). These experimental results allowed the authors to study the range of temperature and pressure conditions where Fischer-Tropsch catalysis proceeds effectively in the Saturnian subnebula. Using a stationary high-pressure subnebular model (Prinn \& Fegley 1989), they concluded that $\mathrm{CH}_{4}$-rich satellitesimals could be formed in the catalytically-active region of the subdisk and thus may have played an important role in the origin of Titan's atmosphere. However, in the context of a time-dependent accretion subdisk model, which provides a more realistic description of the subnebula's evolution than stationary models ${ }^{1}$, FischerTropsch catalysis may not lead efficiently to the formation of

1 Time-dependent subdisk accretion models are fully compatible with the standard giant planet core-accretion formation scenario (Pollack et al. 1996; Magni \& Coradini 2004; Alibert et al. 2005a).
$\mathrm{CH}_{4}$-rich satellitesimals. Indeed, even if pressure and temperature conditions are initially high enough to permit rapid production of $\mathrm{CH}_{4}$ in a heterogeneous gas-phase, the accretion of the subnebula's material onto the giant planet may be faster than the cooling time needed to trap $\mathrm{CH}_{4}$ in satellitesimals formed in the subdisk.

In this work, we examine the $\mathrm{CH}_{4}$ production via FischerTropsch catalysis in the Jovian subnebula and its implications for the composition of satellitesimals by using an evolutionary turbulent model developed by Alibert et al. (2005a). The evolution of this model is divided in two phases: an initially dense and warm phase during which the subnebula is fed from its outer edge by material accreted from the solar nebula and a cold second phase starting at the disappearance of the solar nebula and whose lifetime corresponds to the time required for the subdisk to empty. The strategy describing the choice of the different subdisk parameters is described in Alibert et al. (2005a), and the different parameters of the Jovian subnebula are recalled in Table 1. The viscosity parameter $\alpha$ of the subdisk has been chosen in order to be compatible with the present day orbital distribution of Jupiter's satellites from type I migration calculations performed during their formation. The initial accretion rate and radius of the subdisk have been constrained by models of Jupiter's formation (Alibert et al. 2005b; Magni \& Coradini 2004).

From this model, Mousis \& Alibert (2006) proposed that regular icy satellites may have formed from satellitesimals 
Table 1. Thermodynamic parameters of the Jovian subnebula developed by Alibert et al. (2005a).

\begin{tabular}{lc}
\hline \hline $\begin{array}{l}\text { Thermodynamic } \\
\text { parameters }\end{array}$ \\
\hline Mean mol. weight $(\mathrm{g} /$ mole $)$ & 2.4 \\
Viscosity parameter $\alpha$ & $2 \times 10^{-4}$ \\
Initial disk's radius $\left(R_{J}\right)$ & 150 \\
Initial disk's mass $\left(M_{J}\right)$ & $3 \times 10^{-3}$ \\
Initial accretion rate $\left(M_{J} / \mathrm{yr}\right)$ & $9 \times 10^{-7}$ \\
\hline
\end{tabular}

produced either in the solar nebula or in the subdisk. They also concluded that the chemical composition of ices incorporated in regular satellites is nearly identical in both formation scenarios, due to the inhibition of the homogeneous gas-phase chemistry in the Jovian subnebula.

However, heterogeneous gas-phase chemistry was not taken into account in the afore-mentioned study. Therefore, we investigate here in which part of the Jovian subnebula Fischer-Tropsch catalysis may have been efficient and then determine to what extent the composition of satellitesimals produced in situ may be different from that of solids formed in the solar nebula.

The outline of the paper is as follows. In Sect. 2, we describe the Fischer-Tropsch catalysis in the pressure range of interest. In Sect. 3, we first investigate the possible existence of a catalytically-active region in the Jovian subnebula. We then evaluate if, during the cooling of the subnebula, the produced methane can be preserved in the gas-phase up to its trapping in the forming ices. Section 4 is devoted to discussion.

\section{Fischer-Tropsch catalysis}

At low pressures $\left(2 \times 10^{-2}-5 \times 10^{-1}\right.$ bar $)$ and high $\mathrm{H}_{2} / \mathrm{CO}$ and $\mathrm{H}_{2} / \mathrm{CO}_{2}$ ratios $(=1000)$, the region of efficient conversions of $\mathrm{CO}$ into $\mathrm{CH}_{4}$ and of $\mathrm{CO}_{2}$ into $\mathrm{CH}_{4}$ via Fischer-Tropsch catalysis is narrow in a subnebula $(T=\sim 550 \mathrm{~K})$, according to laboratory experiments (Sekine et al. 2005). This is because the surface of catalytic iron grain is poisoned at temperatures above $600 \mathrm{~K}$ (poisoning is a loss of catalytic activity through conversion of surface carbide to unreactive carbon). The experimental results show that the $\mathrm{CH}_{4}$ formation rates from $\mathrm{CO}$ and $\mathrm{CO}_{2}$ at $550 \mathrm{~K}$ increase with pressure and show similar pressure dependence, but the $\mathrm{CH}_{4}$ formation rates of $\mathrm{CO}_{2}$ are about 0.01 times those of $\mathrm{CO}$ for same reaction conditions. This indicates that $\mathrm{CO}$ is converted into $\mathrm{CH}_{4}$ via Fischer-Tropsch catalysis about 100 times faster than $\mathrm{CO}_{2}$ in the thermodynamic conditions mentioned above.

Using the experimental data on $\mathrm{CH}_{4}$ formation rates, timescales for the conversions of $\mathrm{CO}$ into $\mathrm{CH}_{4}\left(t_{\mathrm{CO} \rightarrow \mathrm{CH}_{4}}\right)$ and of $\mathrm{CO}_{2}$ into $\mathrm{CH}_{4}\left(t_{\mathrm{CO}_{2} \rightarrow \mathrm{CH}_{4}}\right)$ at $550 \mathrm{~K}$ are estimated as a function of pressure in the gas-phase conditions of the solar nebula (Fig. 1). We assume that the gas-phase abundances of elements in the Jovian subnebula are similar to those in the nebula ${ }^{2}$. Note that, since measurements by Sekine et al. (2005) were conducted in the $10^{-2}-10^{-1}$ bar pressure region, values in a lower pressure range have been extrapolated using a linear fit (see Fig. 5 of Sekine et al. 2005). Sekine et al. (2005) showed the clear relationship between the $\mathrm{CH}_{4}$ formation rate and total gas pressure in the subnebula in their experiments. Although

\footnotetext{
2 This assumption is reasonable since the subnebula is fed from its outer edge by gas and gas-coupled solids originating from the solar nebula during the first phase of its evolution (Alibert et al. 2005a).
}

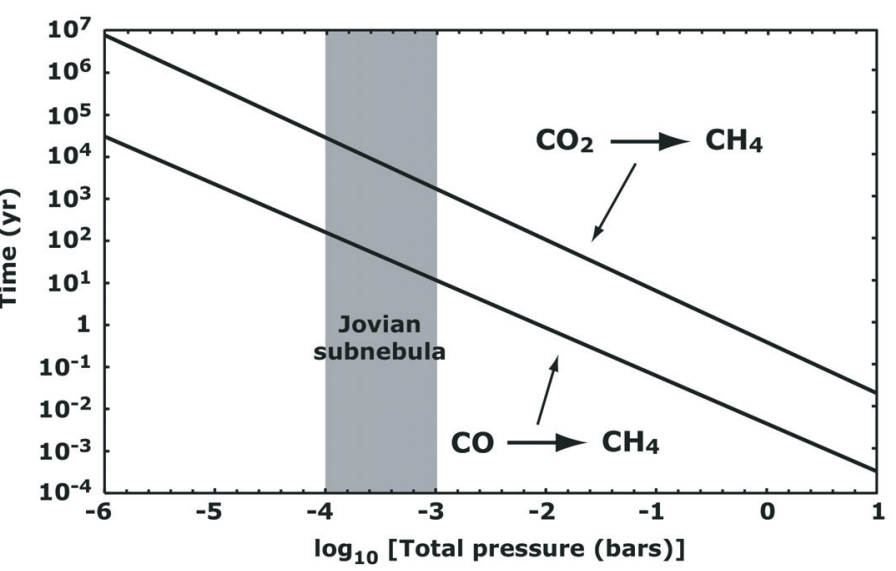

Fig. 1. Timescales for the conversions of $\mathrm{CO}$ into $\mathrm{CH}_{4}$ and of $\mathrm{CO}_{2}$ into $\mathrm{CH}_{4}$ at a temperature of $550 \mathrm{~K}$ as a function of pressure in the gasphase conditions described in Sect. 2. The grey area corresponds to the pressure range encountered in the Jovian subnebula at a temperature of $550 \mathrm{~K}$, where Fischer-Tropsch catalysis is the most efficient (see text).

the experimental conditions have higher pressures than the realistic subnebular pressure, we believe that this relation is valid in the pressure range of our subnebula model. We also assume that about $10 \%$ of the cosmic component of iron was present as metal in the solar nebula (Pollack et al. 1994) and that the particles were spherical with radii of $10^{-6} \mathrm{~m}$ (Sekine et al. 2005). The number densities of $\mathrm{CO}$ and $\mathrm{CO}_{2}$ are deduced assuming solar composition (Anders \& Grevesse 1989), taking all carbon atoms to be in the form of $\mathrm{CO}$ and $\mathrm{CO}_{2}$, respectively. This assumption gives an upper estimate for the timescales for the conversions of $\mathrm{CO}$ and $\mathrm{CO}_{2}$ under the conditions of subnebula. From the evolutionary turbulent model of the Jovian subnebula developed by Alibert et al. (2005a), the pressure range encountered in the Jovian subnebula at $550 \mathrm{~K}$ is calculated to be about $10^{-4}$ to $10^{-3}$ bar. This pressure range is still high enough for $\mathrm{CH}_{4}$ to dominate $\mathrm{CO}$ under chemical equilibrium. In this pressure range, if transport and cooling processes are not taken into account in the Jovian subnebula, $\mathrm{CO}$ and $\mathrm{CO}_{2}$ are entirely converted into $\mathrm{CH}_{4}$ via Fischer-Tropsch catalysis in about $10^{1}-10^{2}$ and $10^{3}-10^{4}$ years, respectively.

\section{Implications for the composition of ices formed in the Jovian subnebula}

Fischer-Tropsch catalysis is efficient up to the distance of $65 R_{\mathrm{Jup}}$ (Jupiter radii) in the early Jovian subnebula since temperatures above $550 \mathrm{~K}$ are not reached at larger distances from the giant planet (see Fig. 6 of Alibert et al. 2005a). Figure 2 gives $t_{\mathrm{CO} \rightarrow \mathrm{CH}_{4}}$ and $t_{\mathrm{CO}_{2} \rightarrow \mathrm{CH}_{4}}$ calculated as a function of the distance from Jupiter in the subnebula and at a local temperature of $550 \mathrm{~K}$ in the gas phase. One must note that these chemical conversion times correspond to different epochs in the subnebula's evolution. This is due to the progressive cooling of the subnebula, which makes the catalytically-active zone move inwards with time from $65 R_{\text {Jup }}$ to the inner edge of the subdisk. This figure also illustrates the pressure dependence of the chemical timescales in the Jovian subnebula. Indeed, at a temperature of $550 \mathrm{~K}$, the gas pressure reduces with increasing distance from Jupiter, thus increasing $t_{\mathrm{CO} \rightarrow \mathrm{CH}_{4}}$ and $t_{\mathrm{CO}_{2} \rightarrow \mathrm{CH}_{4}}$.

Figure 3 shows the timescale $t_{\text {viscous }}$ required by an element of the subdisk to accrete onto Jupiter as a function of the 


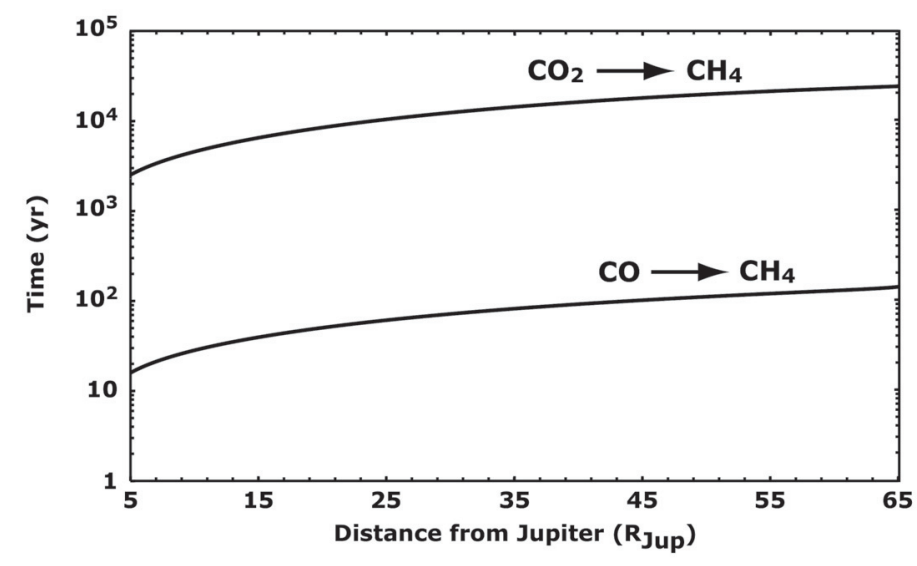

Fig. 2. Timescales for the conversions of $\mathrm{CO}$ into $\mathrm{CH}_{4}$ and of $\mathrm{CO}_{2}$ into $\mathrm{CH}_{4}$ at a temperature of $550 \mathrm{~K}$ in the subnebula as a function of the distance from Jupiter.

planetary separation and when the local temperature is $550 \mathrm{~K}$. This momentum diffusion timescale, resulting from the general theory of viscous disks, is given by $t_{\text {viscous }}=2 r^{2} / 3 v$ where $r$ and $v$ are the distance from Jupiter and the turbulent viscosity in the subnebula given by the model of Alibert et al. (2005a). This figure also represents the timescale ( $\Delta t$ in Fig. 3) required for the subdisk to cool down from $550 \mathrm{~K}$ to the ice condensation temperature that is assumed here to be $150 \mathrm{~K}$ (approximately the crystallization temperature of water ice) as a function of the distance from Jupiter. For a given planetary separation, the two timescales are calculated at the same epoch of the subnebula's evolution. The closer the distance of calculations from Jupiter, the more evolved the subnebula.

It can be seen that, at a given planetary separation, $t_{\mathrm{CO} \rightarrow \mathrm{CH}_{4}}$ is shorter than $t_{\text {viscous }}$, whereas $t_{\mathrm{CO}_{2} \rightarrow \mathrm{CH}_{4}}$ is longer than $t_{\text {viscous. This }}$ implies that only $\mathrm{CO}$ can be completely converted into $\mathrm{CH}_{4}$ via Fischer-Tropsch catalysis in our evolving model of the Jovian subnebula, the conversion of $\mathrm{CO}_{2}$ into $\mathrm{CH}_{4}$ being much less efficient.

In order to determine if the resulting methane can be incorporated in ices formed in the Jovian subnebula, $t_{\text {viscous }}$ must be compared with $\Delta t$. From Fig. 3 , it can be seen that $\Delta t$ is still at least two orders of magnitude higher than $t_{\text {viscous }}$ in the zone of the Jovian subnebula where Fischer-Tropsch catalysis is efficient. This implies that any methane produced in the catalyticallyactive zone will be accreted onto Jupiter long before having been incorporated into the forming icy grains. Moreover, note that values of $\Delta t$ should be higher than those used in this work since volatiles are trapped as hydrates or clathrate hydrates at lower temperatures than $150 \mathrm{~K}$ in the Jovian subnebula (Mousis $\&$ Alibert 2006). From these calculations, we can infer that in an evolving turbulent subnebula, even if Fischer-Tropsch catalysis is active, it has no influence on the composition of the forming microscopic icy grains. These icy grains rapidly agglomerate, decouple from gas, and form satellitesimals. This result does not depend on any specific satellite formation mechanism because the Fischer-Tropsch products are not cooled down at low enough temperatures to allow them being trapped in icy grains; they are just transported toward the giant planet.

Because of this, volatiles incorporated in the satellitesimals formed in the catalytically-active region of the Jovian subnebula must have been provided by the solar nebula rather than being produced via Fischer-Tropsch catalysis in the subdisk.

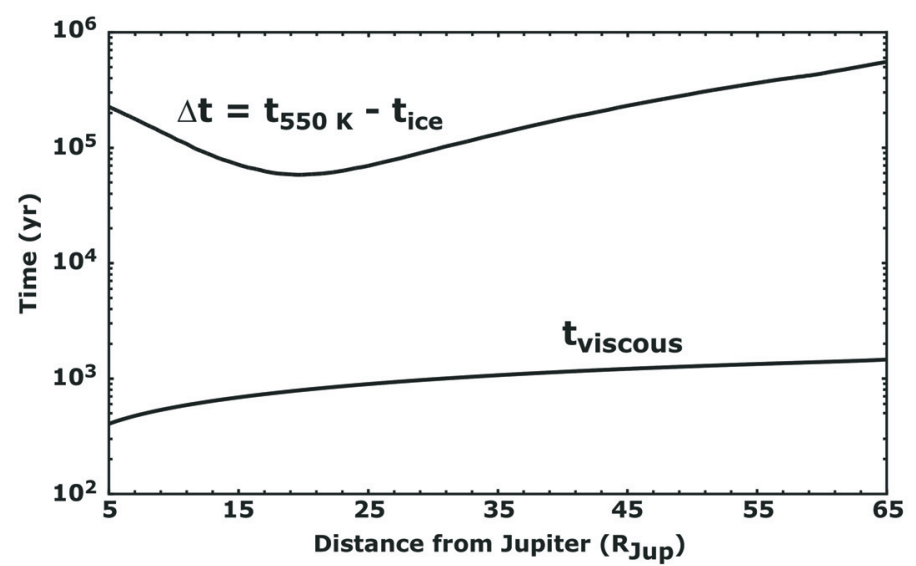

Fig. 3. From top to bottom: time needed by the subnebula to cool down from $550 \mathrm{~K}$ to the condensation temperature of water ice $(150 \mathrm{~K})$ and the viscous timescale of the subdisk as a function of the distance from Jupiter.

\section{Discussion}

This study did not take into account the outward diffusive transport of $\mathrm{CH}_{4}$ initially produced in the catalytically-active region of the subnebula. On the other hand, the advection of gas and gas-coupled solids is the dominant transport mechanism during the first phase of the subnebula's evolution since about $25 \%$ of Jupiter's mass has yet to be accreted through the subdisk (Alibert et al. 2005a). Moreover, if some radial mixing occurred in the subnebula, the diffused $\mathrm{CH}_{4}$ would mix with the other carbon compounds of the gas-phase. Since $\mathrm{CO}$ and $\mathrm{CO}_{2}$ are expected to be much more abundant than $\mathrm{CH}_{4}$ in the material accreted from the solar nebula by the Jovian subnebula ${ }^{3}$, the resulting composition of satellitesimals produced in the outer subnebula during its cooling would still incorporate large quantities of $\mathrm{CO}$ and $\mathrm{CO}_{2}$ and would then be close to that of solids formed in the solar nebula. Therefore, we conclude that the presence of a catalytically-active region within our evolutionary model of the Jovian subnebula does not favor the in situ formation of $\mathrm{CH}_{4}$-rich and $\mathrm{CO}-\mathrm{CO}_{2}$-poor planetesimals, in contrast with the expectation of Sekine et al. (2005) from stationary models. As a result, the composition of ices included in the Jovian regular icy satellites formed from satellitesimals, either produced in the subnebula or in the solar nebula, should be similar. The composition of Jovian regular satellite ices calculated by Mousis \& Alibert (2006) thus remains valid.

In this work, we have used the Jovian subnebula model elaborated by Alibert et al. (2005a). The outer radius of the subdisk and the accretion rate from the nebula have been fixed using recent models of Jupiter formation (Magni \& Coradini 2004; Alibert et al. 2005b). This implies that the structure of the Jovian subnebula only depends on the viscosity parameter $\alpha$. We are aware that the use of a $\alpha$-turbulent model is a simplified approximation to describe the thermodynamic evolution of the Jovian subnebula. In particular, little is known about the possible mechanisms responsible for the turbulent transport of angular momentum within the subnebula. As a result, turbulent viscosity may vary as a function of time and the distance from Jupiter within the subdisk. On the other hand, from migration calculations in

${ }^{3} \mathrm{CO}_{2}: \mathrm{CO}: \mathrm{CH}_{4}$ may vary between 10:10:1 and 40:10:1 in the gasphase of Jupiter's feeding zone (Alibert et al. 2005b; Mousis \& Alibert 2006). 
the subnebula, Alibert et al. (2005a) found that the $\alpha$-parameter should range between $2 \times 10^{-4}$ and $\sim 10^{-3}$ in order to be compatible with the present-day structure of Jupiter's satellite system. Since the value of $\alpha$ adopted in our calculations is the lowest permitted one, we tested the influence of an increase in this parameter on the heterogeneous gas-phase chemistry. In that case, assuming $\alpha=10^{-3}, \Delta t$ is still $\sim$ two orders of magnitude higher than $t_{\text {viscous }}$ in the catalytically-active region of the subnebula. Consequently, we infer that Fischer-Tropsch catalysis still has no influence on the composition of planetesimals formed in a Jovian subnebula with a higher value for the viscosity parameter. Moreover, note that we assumed a disk opacity that is not influenced by the growth of icy grains during the cooling of the subnebula. In reality, the growth of icy grains should significantly reduce the disk opacity, which may potentially speed up the cooling of the Jovian subnebula.

Calculations of the efficiency of Fischer-Tropsch catalysis in the Jovian subnebula require the use of time-dependent subdisk models. Comparisons of the present work with stationary models of the Jovian subnebula are then inappropriate. Conclusions similar to those presented in this study were obtained with the evolutionary Jovian subdisk model of Mousis \& Gautier (2004) (the only other time-dependent accretion disk model ever published, to the best of our knowledge). In that case, the catalyticallyactive zone of the subdisk is even twice smaller than the present model (maximum extent of 30 Jupiter radii). However, the model of Mousis \& Gautier (2004) does not fully agree with the actual scenarios of subnebula formation (Lubow et al. 1999; Magni \& Coradini 2004). In particular, this model behaves as a close system, neglecting the fact that the giant planet continues to accrete material from the solar nebula during a substantial fraction of time.

The presence of a catalytically-active region in the Jovian subnebula also implies that methane and water formed via Fischer-Tropsch catalysis are accreted with the gas onto theforming Jupiter. However, this process has no implications for the current composition of Jupiter's atmosphere since, whatever the initial form of the carbon compound delivered with the gas phase to the forming giant planet, its current atmosphere remains dominated by $\mathrm{H}_{2}, \mathrm{He}, \mathrm{CH}_{4}, \mathrm{H}_{2} \mathrm{O}$, and $\mathrm{NH}_{3}$, due to the high temperatures encountered at very high pressure depths.

Finally, we note that the conclusions of this study also apply to the formation of satellitesimals in Saturn's subnebula. Recent calculations by Alibert \& Mousis (2006) show that Saturn's subnebula displays a thermodynamic structure and evolution similar to that of the Jovian subnebula. This implies that Fischer-Tropsch catalysis did not modify the composition of ices produced in Saturn's subnebula. The methane observed in Titan most likely originates from the solar nebula gas-phase, as suggested by Mousis et al. (2002) for interpreting the D:H ratio in $\mathrm{CH}_{4}$ measured in its atmosphere, rather than having been produced in a catalytically-active region of Saturn's subnebula.

Acknowledgements. This work was supported in part by the Swiss National Science Foundation. We thank Brett Gladman who made interesting suggestions that helped us improve our first manuscript. We are very grateful to the anonymous referee who gave us helpful comments and suggestions.

\section{References}

Alibert, Y., Mousis, O., \& Benz, W. 2005a, A\&A, 439, 1205

Alibert, Y., Mousis, O., \& Benz, W. 2005b, ApJ, 622, L145

Alibert, Y., \& Mousis, O. 2006, In 37th Annual Lunar and Planetary Science Conference, 37,1141

Anders, E., \& Grevesse, N. 1989, Geochim. Cosmochim. Acta, 53, 197

Kress, M. E. \& Tielens, A. G. G. M. 2001, Meteor. \& Planet. Sci., 36, 75

Lubow, S. H., Seibert, M., \& Artymowicz, P. 1999, ApJ, 526, 1001

Magni, G., \& Coradini, A. 2004, Planet. Space Sci., 52, 343

Mousis, O., \& Gautier, D. 2004, Planet. Space Sci., 52, 361

Mousis, O., \& Alibert Y. 2006, A\&A, 448, 771

Mousis, O., Gautier, D., \& Coustenis, A. 2002, Icarus, 159, 156

Pollack, J. B., Hollenbach, D., Beckwith, S., et al. 1994, ApJ, 421, 615

Pollack, J. B., Hubickyj, O., Bodenheimer, P., et al. 1996, Icarus, 124, 62

Prinn, R. G., \& Fegley Jr., B. 1989, In Origin and Evolution of Planetary and Satellites Atmospheres, ed. S. K. Atreya, J. B. Pollack, M. S. Matthews (Tucson: The University of Arizona Press), 78

Sekine, Y., Sugita, S., Shido, T., et al. 2005, Icarus, 178, 154

Willacy, K. 2004, ApJ, 600, 87 